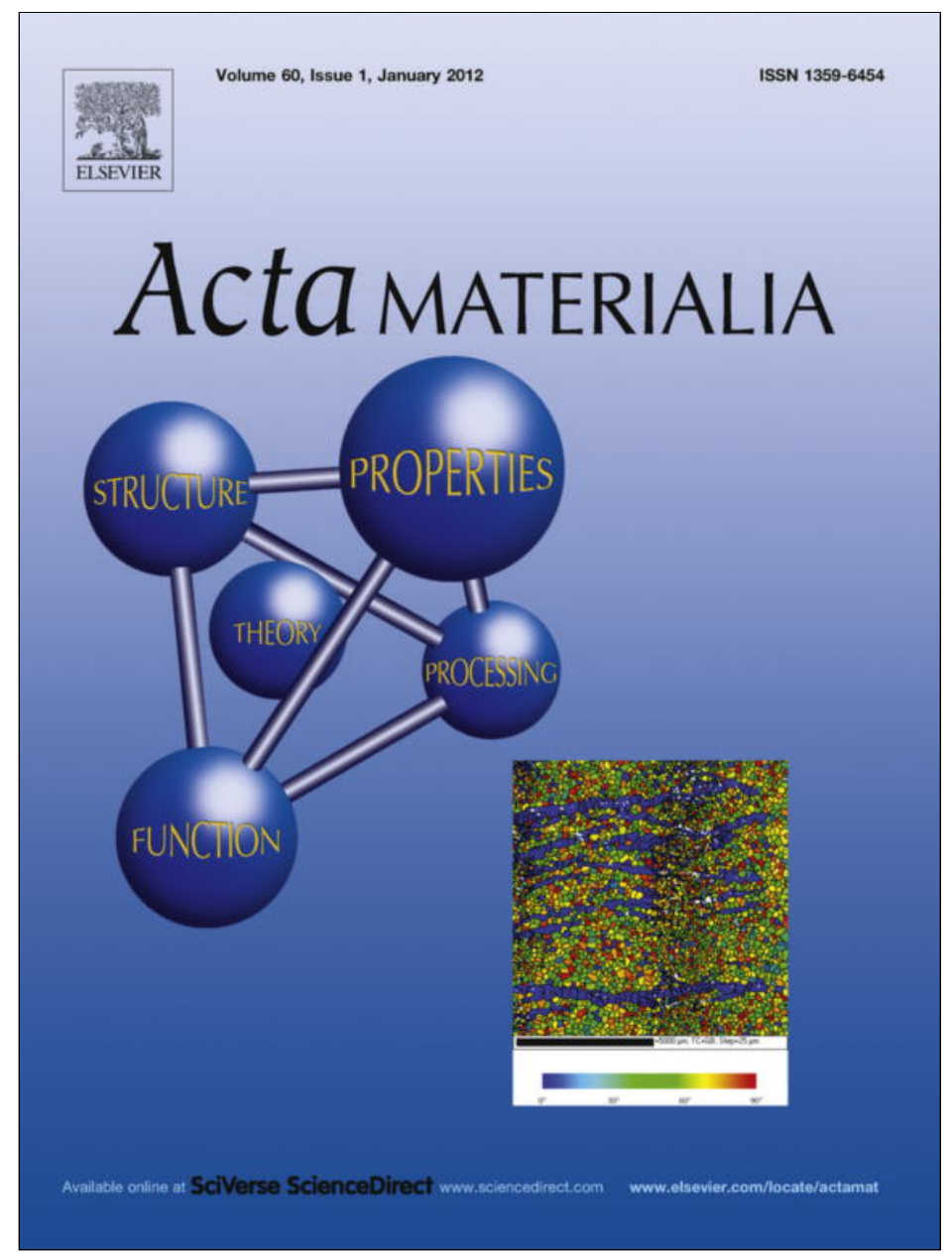

(This is a sample cover image for this issue. The actual cover is not yet available at this time.)

This article appeared in a journal published by Elsevier. The attached copy is furnished to the author for internal non-commercial research and education use, including for instruction at the authors institution and sharing with colleagues.

Other uses, including reproduction and distribution, or selling or licensing copies, or posting to personal, institutional or third party websites are prohibited.

In most cases authors are permitted to post their version of the article (e.g. in Word or Tex form) to their personal website or institutional repository. Authors requiring further information regarding Elsevier's archiving and manuscript policies are encouraged to visit: 


\title{
Three-dimensional granular model of semi-solid metallic alloys undergoing solidification: Fluid flow and localization of feeding
}

\author{
M. Sistaninia ${ }^{\mathrm{a}, *}$, A.B. Phillion ${ }^{\mathrm{b}}$, J.-M. Drezet ${ }^{\mathrm{a}}$, M. Rappaz ${ }^{\mathrm{a}}$ \\ ${ }^{a}$ Computational Materials Laboratory, Ecole Polytechnique Fédérale de Lausanne, Lausanne, Switzerland \\ ${ }^{\mathrm{b}}$ Okanagan School of Engineering, University of British Columbia, Kelowna, BC, Canada
}

Received 3 February 2012; received in revised form 19 March 2012; accepted 19 March 2012

\begin{abstract}
A three-dimensional (3-D) granular model which simulates fluid flow within solidifying alloys with a globular microstructure, such as that found in grain refined Al alloys, is presented. The model geometry within a representative volume element (RVE) consists of a set of prismatic triangular elements representing the intergranular liquid channels. The pressure field within the liquid channels is calculated using a finite elements (FEs) method assuming a Poiseuille flow within each channel and flow conservation at triple lines. The fluid flow is induced by solidification shrinkage and openings at grain boundaries due to deformation of the coherent solid. The granular model predictions are validated against bulk data calculated with averaging techniques. The results show that a fluid flow simulation of globular semi-solid materials is able to reproduce both a map of the 3-D intergranular pressure and the localization of feeding within the mushy zone. A new hot cracking sensitivity coefficient is then proposed. Based on a mass balance performed over a solidifying isothermal volume element, this coefficient accounts for tensile deformation of the semi-solid domain and for the induced intergranular liquid feeding. The fluid flow model is then used to calculate the pressure drop in the mushy zone during the direct chill casting of aluminum alloy billets. The predicted pressure demonstrates that deep in the mushy zone where the permeability is low the local pressure can be significantly lower than the pressure predicted by averaging techniques.
\end{abstract}

(C) 2012 Acta Materialia Inc. Published by Elsevier Ltd. All rights reserved.

Keywords: Solidification; Microstructure; Liquid feeding; Finite elements modeling; Hot tearing

\section{Introduction}

During solidification the feeding of liquid to counteract solidification shrinkage is hindered by the morphology and dense packing of the grain structure. When such regions within a casting are also submitted to tensile thermal deformation a solidification defect known as hot tearing [1-4] or solidification cracking may develop. In industrial metallic alloys direct observation of the semi-solid microstructure is challenging due to the high temperatures and metal opacity. While in situ observations of model organic systems [5] and small metallic specimens [6] subject to tensile loading are now available, accurate modeling of the localization

\footnotetext{
* Corresponding author. Tel.: +41 216933943.

E-mail address: meisam.sistaninia@epfl.ch (M. Sistaninia).
}

of liquid feeding has become a prerequisite for predicting hot tear formation.

Accurate simulations of liquid convection during the solidification of industrially cast components is challenging since the typical length scale of the liquid network, i.e. the grain size, is usually much smaller than the process dimension. In order to overcome this issue early researchers used an averaging or a multiphase mixture approach on the macro-scale to investigate fluid flow within the mushy zone [7-11]. In this type of simulation a representative volume element (RVE) methodology is used with the RVE assumed to consist of only one "average phase" based on a combination of solid and liquid properties. The RVE is large with respect to the grain size yet small with respect to the process scale. The governing equations are derived from averaging techniques in which the concepts of the 
volume fractions of solid and liquid as well as exchange terms at the solid-liquid interface are introduced. More specifically, for fluid flow occurring in the mushy zone Darcy's law, which describes the flow of fluid through a porous medium, is often used to link the physical structure with fluid flow. This equation relates the average (or superficial) intergranular velocity to the pressure gradient, with a parameter that is proportional to the permeability of the mushy zone and inversely proportional to the dynamic viscosity $[12,13]$.

Although the averaging approach has been successful in providing an insight into porosity formation, hot tearing and macrosegregation, it does not provide any information at the level of the microstructure. Thus important physical phenomena, such as feeding variations at the level of the grains, cannot be obtained to improve hot tearing predictions. Over the past few years a new technique based on granular modeling has shown much promise for investigating the initiation and propagation of cracks within liquid films [14-23]. In this technique the liquid and solid phases are modeled independently but with a large enough domain size to be considered a RVE.

Early solidification models based on a granular modeling approach considered a regular arrangement of grains for the simulation of liquid feeding within mushy zones [17]. However, such models have a key shortcoming associated with the intrinsic specific solid-liquid interfacial area $A_{s \ell}$, i.e. the area of the solid-liquid interfacial area normalized to the volume of the solid. In a regular arrangement of grains all the solid grains solidify at the same rate and therefore all connect at the same time when the overall volumetric solid fraction $\left(g_{\mathrm{s}}\right)$ within the RVE reaches unity. Thus the area $A_{s \ell}$ continuously increases until $g_{\mathrm{s}}=1$. In reality, however, the random arrangement of the grains and thus their different sizes result in the formation of clusters (bridging or coalescence of neighboring grains) of increasing size, prior to $g_{\mathrm{s}}$ reaching unity. The process of coalescence in a random array of grains typically occurs once $g_{\mathrm{s}}>0.9$ [24] and causes $A_{s \ell}$ to reach a maximum at $g_{\mathrm{s}}<1$. As first proposed in two dimensions by Mathier et al. [14], a regular arrangement of grains can be replaced by a random distribution of nucleation centers combined with a Voronoi tessellation. Using this approach a twodimensional (2-D) granular model of fluid flow during equiaxed-globular solidification was then developed by Vernède et al. $[18,21]$ in order to examine liquid feeding during the solidification and semi-solid deformation of an Al-1 wt.\% $\mathrm{Cu}$ alloy. In a subsequent work [19] it was shown that a finer grain structure has a decreased hot tearing sensitivity due to a more evenly distributed flow within more liquid channels, and thus a better feeding of areas under tensile stress.

Although the idea of a random network of grains provides significant improvements in our ability to model fluid flow using a granular approach, the 2-D geometry remains a severe limitation, since granular microstructures are inherently three-dimensional (3-D). Issues include (i) the
$A_{s \ell}$ predicted by a $2-\mathrm{D}$ simulation is smaller than reality [16] and, hence, the semi-solid permeability cannot be accurately quantified, and (ii) although simultaneous continuity of both the solid and liquid phases can exist in three dimensions, a topological feature of 2-D geometry is that only one of the two phases can percolate through the RVE.

In the present work a new 3-D fluid flow model for granular semi-solid materials with a geometry based on a Voronoi diagram has been developed. Its purpose is to investigate the localization of liquid feeding in the mushy zone during the solidification of grain refined aluminum alloys and to overcome the limitations of 2-D geometries discussed before. Firstly, the methodology for generating the liquid film network during solidification within the framework of a granular approach is briefly outlined. Secondly, the fluid flow model is described. Thirdly, the model is validated and then used to explore fluid flow during the globular solidification of an aluminum-copper alloy.

\section{Development of a 3-D fluid flow model of the mushy zone}

\subsection{Generation of the semi-solid liquid film geometry and mesh}

The liquid film geometry for the semi-solid fluid flow simulations is created using the 3-D granular solidification model known as GMS-3D [16,23]. In this model it is assumed that the grains are distributed randomly within an RVE of nearly uniform temperature. Providing that the grains nucleate simultaneously, the final grain structure will be nearly globular, with grain boundaries corresponding to a Voronoi tessellation of the random nucleation centers [25]. The RVE used in the present model, shown in Fig. $1 \mathrm{a}$, is a cube $3 \times 3 \times 3 \mathrm{~mm}$ containing 27,000 grains, i.e. a grain size of about $100 \mu \mathrm{m}$. Solidification is approximated within each grain using a microsegregation model with infinite diffusion in the liquid and some back-diffusion in the solid. As the temperature of the RVE is assumed to be uniform, the solute composition of the liquid $C_{\ell}$ is also uniform in a binary alloy and given by the liquidus temperature $C_{\ell}(T)$ of the alloy (any curvature undercooling is neglected). Although the composition of the solid at the solid-liquid interface $C_{s}^{*}$ is also uniform and given by $k C_{\ell}$, where $k$ is the partition coefficient, the solute profile in each grain (or portion of grain) is not uniform as it also depends on the Fourier number, i.e. on the grain size.

Each polyhedral grain derived from the Voronoi tessellation is divided into a set of pyramids having the nucleation center as the summit and the Voronoi facet as the base. The base of the pyramid is subdivided into triangles so as to only have tetrahedral elements that can be used either in solid deformation [23] or fluid flow calculations (see Fig. 2b). Solute exchange between the tetrahedral pyramids is neglected, and as a result the microsegregation model reduces to a onedimensional solidification simulation in spherical coordinates with the solid-liquid interface advancing from the nucleation center to the Voronoi facet. As it is the height 

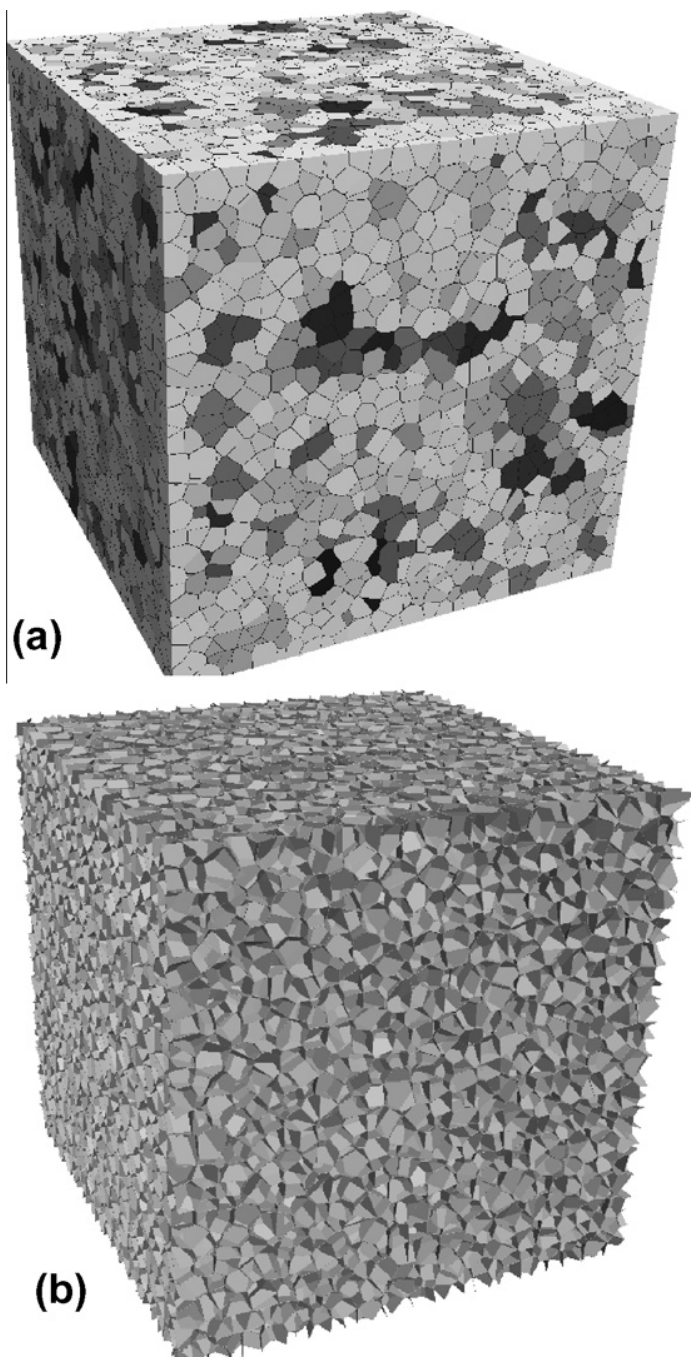

Fig. 1. The semi-solid simulation domain: (a) a partially solidified RVE at $g_{\mathrm{s}}=0.93$, containing $27,000(30 \times 30 \times 30)$ grains; $(\mathrm{b})$ the liquid film network between the polyhedral grains.

$L$ of each pyramid that is entered into the corresponding back-diffusion Fourier number, the pyramids solidify at different solidification velocites $v^{*}$ and coalesce with their neighbors at different times, thus inducing grain percolation and a decrease in $A_{s \ell}$ at $g_{\mathrm{s}}<1$. The solid-liquid interface within each tetrahedron is parallel to the base (future grain boundary), thus subdividing it into a tetrahedral solid and a triangular prismatic liquid channel. At the beginning of solidification the liquid channels are very wide, but as $g_{\text {s }}$ increases the width of the liquid channels is reduced. The coalescence of two neighboring grains is assumed to occur once the liquid film thickness is of the order of the diffuse solid-liquid interface thickness $(1-5 \mathrm{~nm})[14,26]$. In such small channels the permeability of the liquid is very low and the pressure drop required to nucleate a void is very large. Therefore, very thin liquid channels are considered to act in a way similar to solid-solid bonds. To accommodate this physical behavior, grain coalescence is assumed to occur once the thickness of a liquid channel shrinks to $5 \mathrm{~nm}$ in width. This phenomenon leads to the formation of grain clusters (a group of solid grains which are in mechanical contact). In Fig. 1a the grains belonging to the same cluster are shaded the same gray level. Further details of this model, together with a discussion of its limitations and domain of validity, are given in Phillion et al. [16] and Sistaninia et al. [23].

The fluid flow simulation domain, or mesh, accounts for all channels between grains that have not yet coalesced, i.e. all channels with a thickness larger than $5 \mathrm{~nm}$. Flow is assumed to occur in these interconnected intergranular regions only, and not through the grains, as could be the case in dendritic specimens. The mesh consists of triangular prismatic elements (wedges with five facets), as shown in Fig. 2a, with connectivity provided by GMS-3D. As can be seen in Fig. 2b, flow conservation holds at each triple line, i.e. where three different liquid wedges meet. The fluid flow mesh shown in Fig. $1 \mathrm{~b}$ contains $1.03 \times 10^{6}$ elements.

\subsection{Liquid feeding model}

Under the solidification conditions presented above Navier-Stokes equations for fluid flow can be simplified to Poiseuille flow between two parallel plates if one considers the specific geometry of the interconnected liquid channels that remain between the solidifying polyhedral grains and assumes that flow is both parallel to the triangular facet within each element (i.e. the fluid velocity $\vec{v}_{\ell}$ has only two components, $\vec{v}_{\ell x^{\prime}}$ and $\vec{v}_{\ell y^{\prime}}$, in a local frame $\left(x^{\prime}, y^{\prime}, z^{\prime}\right)$ attached to the facet with the local $z^{\prime}$-axis perpendicular to the facet surface), and is irrotational (i.e. the vorticity $\vec{\omega}_{l}=\nabla \times \vec{v}_{\ell}$ is nil)

$\vec{v}_{\ell}=\frac{1}{2 \mu} \nabla p\left[z^{\prime^{2}}-h^{2}\right]$

where the liquid channel width is $2 h, \mu$ is the dynamic viscosity, $p$ the liquid pressure and the reference position for the $z^{\prime}$-axis is placed at mid-distance between the two parallel solid-liquid interfaces of two neighbor grains.

Mass conservation within the liquid film must take into account both solidification shrinkage and deformation of the solid. Considering Fig. 2, if the velocity of the solidliquid interface is $v^{*}$, the normal velocity of the fluid at the interface required to compensate for solidification shrinkage is given by $\beta v^{*}$, where $\beta=\left(\rho_{s} / \rho_{\ell}-1\right)$ is the shrinkage factor and $\rho_{\ell} \rho_{\mathrm{l}}$, and $\rho_{\mathrm{s}}$ are the densities of the liquid and solid, respectively. Concurrently, deformation of the solid grains can induce variation in the liquid channel width if the normal velocity of the two solid grains, $v_{\mathrm{sz}^{\prime}}^{+}$ and $v_{\mathrm{sz}}^{-}$are different. With these considerations in mind, one can then establish a mass balance over the prismatic volume element $V_{\ell}$ having the axis $z^{\prime}$ as generating line and two solid-liquid interfacial areas $S_{s \ell}^{e}$ (see Fig. 2). Integrating this equation over $V_{\ell}$ and assuming the liquid to be incompressible (i.e. $\nabla \cdot \vec{v}_{\ell}=0$ ) results in

$\int_{V_{\ell}} \nabla \cdot \vec{v}_{\ell} d V=\int_{S_{s \ell}^{e}} \vec{v}_{\ell} \cdot \vec{n} d S+\int_{S_{\ell}^{e}} \vec{v}_{\ell} \cdot \vec{n} d S$ 


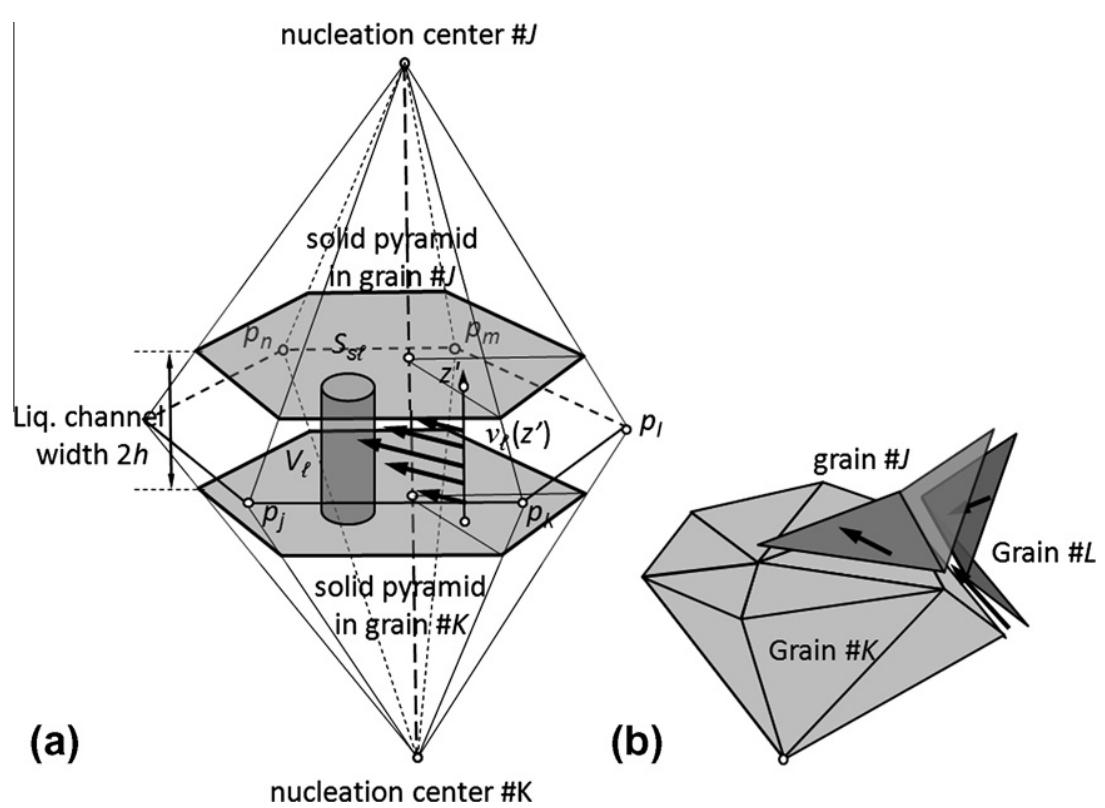

Fig. 2. A schematic of the liquid control volume: (a) its location in relation to the facets of two neighboring grains; (b) the triangular network of liquid elements.

where $S_{\ell}^{e}$ is the lateral surface of the prism and the divergence theorem has been used. At the solid-liquid interface this integral is simply given by:

$\int_{S_{s \ell}^{e}} \vec{v}_{\ell} \cdot \vec{n} d S=S_{s \ell}^{e} v_{\ell n}=S_{s \ell}^{e}\left(2 \beta v_{\ell n}^{*}+\Delta v_{\mathrm{sn}}\right)$

where $\Delta v_{\mathrm{sn}}$ measures the difference $\left(v_{\mathrm{sz}}^{+}-v_{\mathrm{sz}}^{-}\right)$. The integral of the fluid flow velocity along the lateral surface of $V_{\ell}$ is obtained by replacing $\vec{v}_{\ell}$ by Eq. (1). One then has:

$$
\begin{aligned}
\frac{1}{2 \mu} \int_{S_{\ell}^{e}} \nabla p \cdot \vec{n}\left(z^{\prime 2}-h^{2}\right) d z^{\prime} d S & =\frac{-2 h^{3}}{3 \mu} \oint_{\partial S_{\ell}^{e}} \nabla p \cdot \vec{n} d S \\
& =\frac{-2 h^{3}}{3 \mu} \int_{\partial S_{\ell}^{e}} \nabla^{2} p d S=\frac{-2 h^{3}}{3 \mu} \nabla^{2} p S_{s \ell}^{e}
\end{aligned}
$$

Please note that after the integration over $z^{\prime}$ from $-h$ to $+h$ one is left with an integral over the perimeter of $S_{s \ell}^{e}$. Using Green's theorem this integral is equivalent to an integral over the surface $S_{s \ell}^{e}$ of the Laplacian of the pressure field $\nabla^{2} p$, which over a triangular element is constant for a Poiseuille flow. To summarize, as this derivation is valid for any solid-liquid interfacial area $S_{s \ell}^{e}$ one finally has the equation:

$\frac{2 h^{3}}{3 \mu} \nabla^{2} p=2 \beta v^{*}+\Delta v_{s n}$

The left-hand side of Eq. (5) is the net volume of liquid that must flow into the control volume to account for the effects of solidification shrinkage and deformation on fluid flow shown on the right-hand side. The term $2 \beta v^{*}$ is unique for every channel, as it depends on the Fourier number, and is calculated based on the output of the granular solidification model. However, the term $\Delta v_{\text {sn }}$ depends on the strain rate exerted on the mushy zone. In the present work the strain of the solid skeleton is not calculated using a mechanical model [25], but instead it is assumed that the grains are rigid and hence all of the deformation is localized in the liquid channels. In this case $\Delta v_{\text {sn }}$ of each triangular element can be approximated as:

$\Delta v_{\mathrm{sn}}=2 h \frac{\dot{\varepsilon}_{\mathrm{sv}}}{\left(1-g_{\mathrm{s}}\right)}$

where $\dot{\varepsilon}_{\mathrm{sv}}=\dot{\varepsilon}_{\mathrm{xx}}+\dot{\varepsilon}_{\mathrm{yy}}+\dot{\varepsilon}_{\mathrm{zz}}$ is the volumetric part of the strain rate exerted on the mushy zone.

\subsection{Numerical implementation}

A finite element code, written in $\mathrm{C}++$, has been used to calculate the liquid pressure in the semi-solid medium based on Eq. (5). Since the flow within an element has been assumed to be parallel to the facets only, the 3-D prism shaped geometry within which it occurs can be discretized into three node 2-D triangular elements, using the Galerkin method. Please note that in Fig. 2 the polygonal shape of the grain boundary between grains $j$ and $k$ has not been subdivided into triangles in order to make the drawing more readable. In this method the pressure within each triangular element is approximated as:

$p^{e}=\sum_{i=1}^{3} N_{i} p_{i}^{*}$

where $N_{\mathrm{i}}(i=1,2,3)$ are the shape functions of the triangular element that approximate the pressure field within element (e) in the $\left(x^{\prime}, y^{\prime}, z^{\prime}\right)$ coordinate system. The $p_{\mathrm{i}}^{*}$ values $(i=1,2,3)$ define the nodal values of the pressure. Applying the Galerkin finite element method to the governing Eq. (5) the following matrix equation is obtained: 
$[K]^{e}\left\{\begin{array}{l}p_{1}^{*} \\ p_{2}^{*} \\ p_{3}^{*}\end{array}\right\}=b^{e}+\{\phi\}^{e}$

where

$[K]_{i j}^{e}=\frac{2 h^{3}}{3 \mu} \int_{S^{e}}\left(\frac{\partial N_{i}}{\partial x^{\prime}} \frac{\partial N_{j}}{\partial x^{\prime}}+\frac{\partial N_{i}}{\partial y^{\prime}} \frac{\partial N_{j}}{\partial y^{\prime}}\right) d S$

$\{b\}_{i}^{e}=-\int_{S^{e}}\left(2 \beta v^{*}+\Delta v_{s n}\right) N_{i} d S$

$\{\phi\}_{i j}^{e}=\frac{2 h^{3}}{3 \mu} \int_{\partial S^{e}}\left(\frac{\partial N_{j}}{\partial x^{\prime}} n_{x^{\prime}}+\frac{\partial N_{j}}{\partial y^{\prime}} n_{y^{\prime}}\right) p_{j} N_{i} d \Gamma$

Matrix $[K]^{e}$ is the stiffness matrix, $b^{e}$ is the body load associated with solidification shrinkage and solid deformation, $\{\phi\}^{e}$ is the boundary conditions, and $S^{e}$ and $\partial S^{e}$ are the area and perimeter of the triangular element, respectively, while $n=\left(n_{x^{\prime}}, n_{y^{\prime}}\right)$ is the outward pointing unit vector perpendicular to $\partial S^{e}$ in the $\left(x^{\prime}, y^{\prime}, z^{\prime}\right)$ coordinate system (see Fig. 2). The matrix Eq. (8) is then assembled from Eqs. (9)-(11) for each element. Once the individual element matrices have been developed they must be assembled into the global stiffness matrix. Please note that the boundary contributions $\{\phi\}^{e}$ (Eq. (11)) correspond to the intergranular flow leaving (or entering) the perimeter of each element. The boundary condition $\{\phi\}^{\mathrm{e}}$ will remain only on external boundaries of the whole RVE where a Neumann (imposed flux) or Cauchy (mixed) condition is imposed. After developing the global stiffness matrix it is solved with a conjugate gradient linear iterative method using a free open access program $\mathrm{C}++$ template library known as IML $++[27]$.

\section{Results and discussion}

The 3-D fluid flow simulations using the geometry derived from the granular solidification model [16] have been performed on the domain shown in Fig. 1a, unless otherwise stated, under an imposed cooling rate of $-1 \mathrm{~K} \mathrm{~s}^{-1}$. The solidifying material is a binary Al-1 wt.\% $\mathrm{Cu}$ alloy with the following physical parameters: $\rho_{1}=2440 \mathrm{~kg} \mathrm{~m}^{-3}, \mu=1.5 \times 10^{-3} \mathrm{~Pa} \mathrm{~s}$ and $\beta=0.074$. This alloy was chosen for its wide freezing range and susceptibility to hot tear formation [4]. The initial results are presented below, followed by model validation. The model is then used to propose a new hot tearing sensitivity coefficient. The sensitivity coefficient, which similarly to the RDG criterion [4] is based upon a mass balance performed over the liquid and solid phases, accounts for the tensile deformation of the RVE and for the induced intergranular liquid feeding. Finally, the pressure drop within the mushy zone during the direct chill casting of an aluminum alloy extrusion billet is examined.

\subsection{Model validation}

The model has been validated using three comparison methods. First, the assumption of Poiseuille flow is verified.
Second, the permeability of the mushy zone is calculated and compared with the bulk permeability predicted by the Carman-Kozeny relationship [12]. Third, the amount of liquid needed to account for the volumetric change during solidification and semi-solid deformation is calculated and compared with the results obtained from a bulk analysis.

\subsubsection{Solidification geometry}

Fig. 3a shows the distribution of the liquid channel widths predicted by the granular solidification model for three values of $g_{\mathrm{s}}\left(g_{\mathrm{s}}=0.80,0.93\right.$, and 0.98$)$. As solidification proceeds the smaller liquid channels close, while the larger ones remain open due to the effects of back diffusion and the Fourier number in the microsegregation model. As can be seen, the majority of the open channels have a width of $\sim 3 \mu \mathrm{m}$ for $g_{\mathrm{s}}=0.93$, decreasing to $\sim 1 \mu \mathrm{m}$ for $g_{\mathrm{s}}=0.98$. Also, the number of closed channel increases with $g_{\mathrm{s}}$ so that there is an $\sim 40 \%$ increase in the number of closed channels between $g_{\mathrm{s}}=0.93$ and 0.98 . Since the Reynolds number for fluid flow through small narrow channels is very small $\left(\simeq 10^{-5}\right)$ fluid motion can be considered laminar. Fig. $3 b$
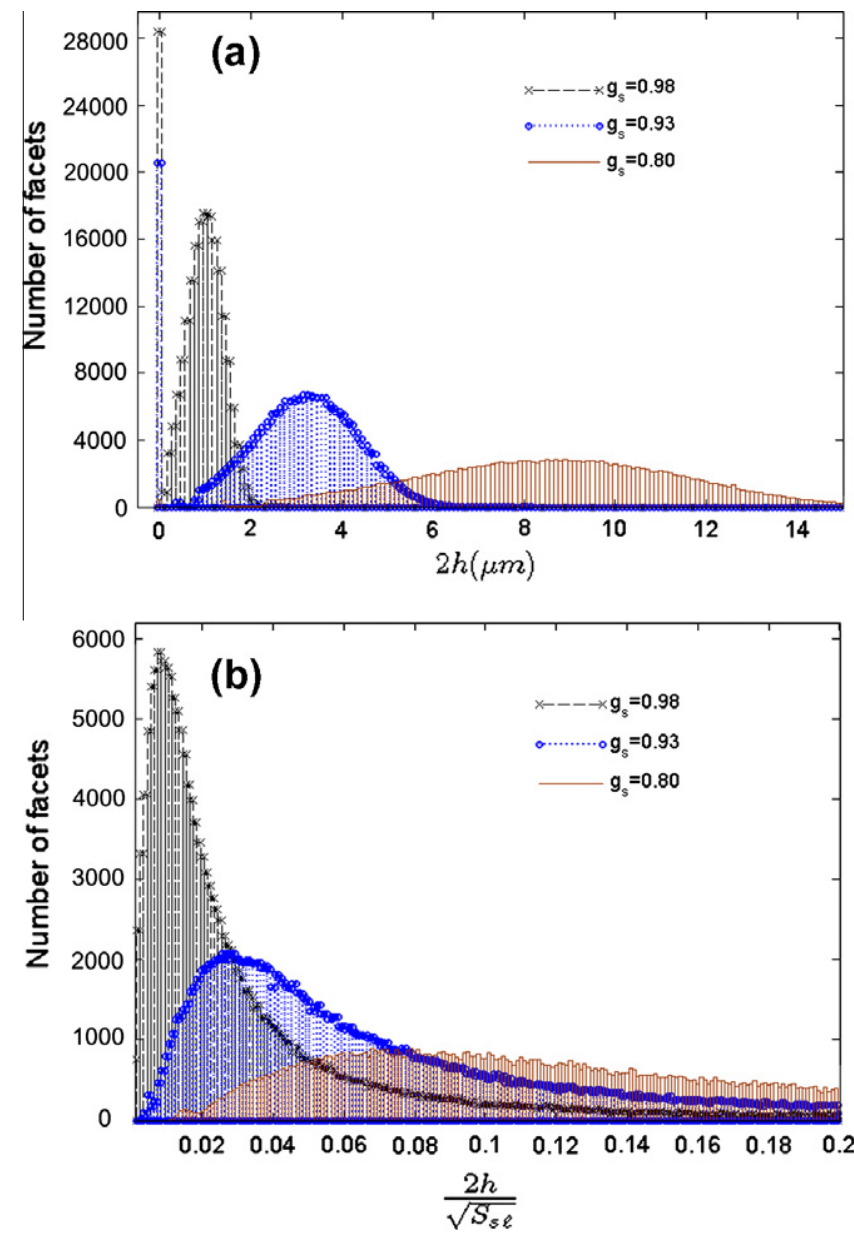

Fig. 3. (a) Distribution of the liquid channel width for three values of $g_{\mathrm{s}}$, including those which are already closed (width $=0$ ). (b) The same distribution but for open channels only and normalized to the square root of $S_{s \ell}$, the surface of each corresponding Voronoi facet. 
shows the distribution of the ratio of the liquid channel thickness to the square root of the surface $S_{s \ell}$ of the corresponding facet of the Voronoi tessellation. As can be seen, the majority of the liquid channels have a width to length ratio of less than $6 \%$ when $g_{\mathrm{s}}>0.90$ and, hence, the channels can be considered infinite parallel plates. Thus the assumption of Poiseuille flow (laminar flow between two infinite parallel plates) is valid for the case $0.9<g_{\mathrm{s}}<1$.

\subsubsection{Permeability}

For semi-solid materials with an equiaxed microstructure, such as grain refined industrial castings susceptible to hot tearing, the bulk permeability $\mathcal{K}$ has often been expressed by the Carman-Kozeny relation [12]:

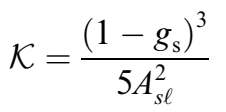

The factor 5 is an empirical fitting factor which seems to provide good agreement with experimental data over a wide range of solid fractions $g_{\mathrm{s}}$ for the case of an isotropic porous medium composed of unconsolidated material $[12,13,28,29]$. In order to assess the ability of the new 3D fluid flow model to predict the bulk permeability a series of isothermal flow simulations were performed at various $g_{\mathrm{s}}$ values in the interval $[0,1]$ with the following boundary conditions: a constant pressure $p_{0}$, i.e. a Dirichlet boundary condition, on the top side and a fixed flux $q$, i.e. a Neumann boundary condition, on the bottom side. Please note that $q$ has units of velocity, since it is a flow $\left(\mathrm{m}^{3} \mathrm{~s}^{-1}\right)$ per unit area $\left(\mathrm{m}^{2}\right)$. Furthermore, for a valid comparison with Carman-Kozeny permeability it is necessary to neglect solidification shrinkage and to close the lateral boundaries. Darcy's law is then used to calculate the permeability using the pressure $p_{1}$ found at the bottom boundary:

$\mathcal{K}=\frac{q \mu}{\left(\frac{P_{1}-P_{0}}{L}\right)}$

where $L$ is the height of the domain and $p_{1}$ is the average pressure on the bottom side of the RVE.

Fig. 4 shows the pressure contours for four values of $g_{\mathrm{s}}$ $\left(g_{\mathrm{s}}=0.50,0.80,0.92\right.$ and 0.98$)$ with $q=50 \mu \mathrm{m} \mathrm{s}^{-1}$ and $p_{0}=0$. In this figure the calculated liquid flow in each facet is represented with a plate of finite thickness proportional to the local flow and normalized by the overall flow (i.e. relative flow intensity). The legend is shown to the right of each image and although the pressure is defined only in the liquid channels, the color is also represented within the grains for visibility. For the two images at lower $g_{\mathrm{s}}$ (Fig. $4 \mathrm{a}$ and b) the fluid flow is fairly well distributed in the liquid channels. However, the flow appears to localize along a few preferential paths at $g_{\mathrm{s}}=0.92$ (Fig. 4c) and even more at $g_{\mathrm{s}}=0.98$ (Fig. $4 \mathrm{~d}$ ). Also, note that the maximum local pressure drop $\left(p_{0}-p_{1}\right)$ increases with $g_{\mathrm{s}}$; at $g_{\mathrm{s}}=0.98$ it is approximately four orders of magnitude larger than its value for $g_{\mathrm{s}}=0.50$.

Fig. 5 shows a comparison for the bulk semi-solid permeability using the new 3-D fluid-flow model (Fig. 5a), a previously developed 2-D granular fluid-flow model $[18,21]$ (Fig. 5b) and the predictions of the Carman-Kozeny relation (Fig. 5c). Note that the results for $g_{\mathrm{s}}<0.3$ have not been presented since at low $g_{\mathrm{s}}$ the liquid channels are wide and therefore the Poiseuille laminar flow assumption
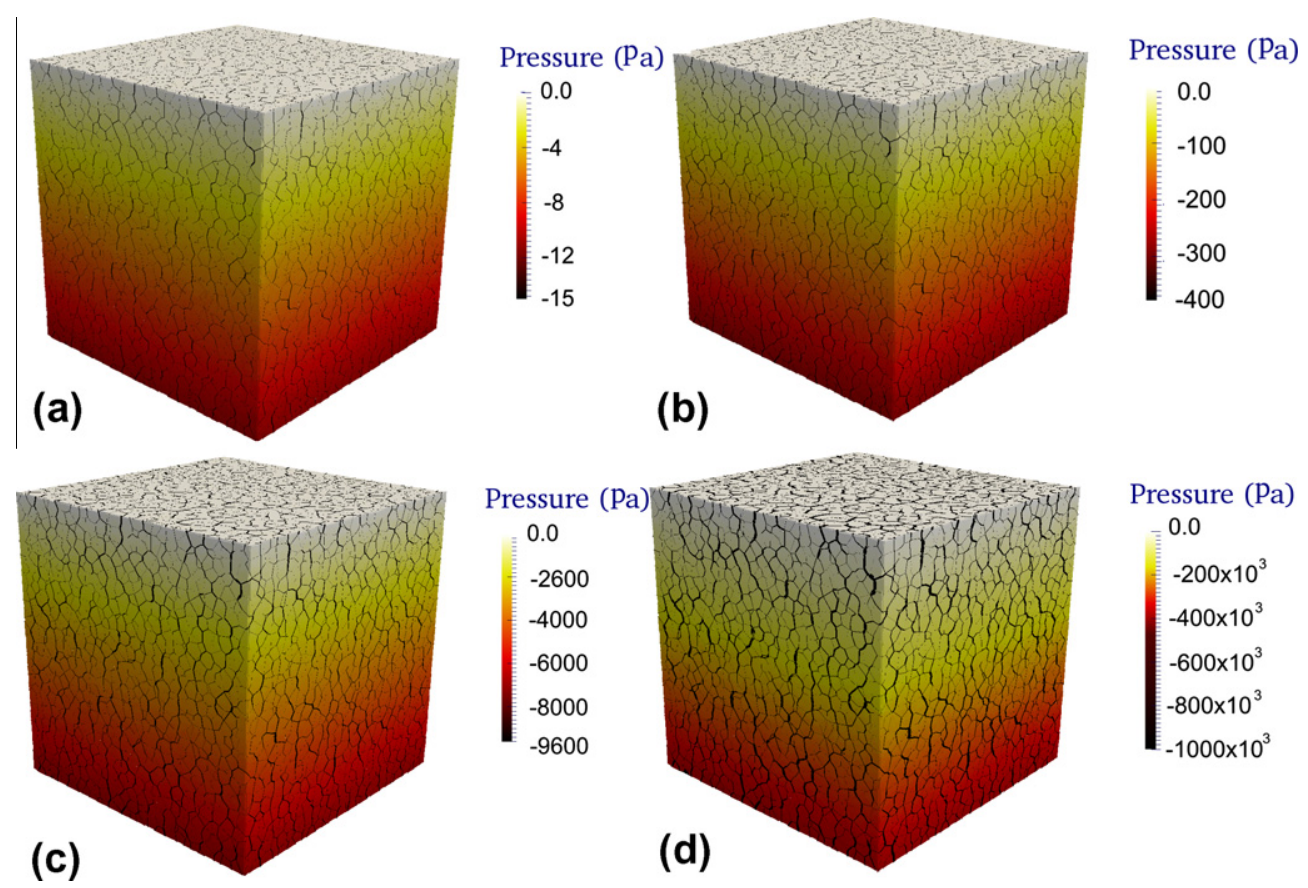

Fig. 4. Fluid flow through a mushy zone volume element $(3 \times 3 \times 3 \mathrm{~mm})$ consisting of 27,000 grains for (a) $g_{\mathrm{s}}=0.5$, (b) $g_{\mathrm{s}}=0.80$, (c) $g_{\mathrm{s}}=0.93$ and $(\mathrm{d})$ $g_{\mathrm{s}}=0.98$. The width of each channel is proportional to the local flow normalized to the total flow within the volume element. 


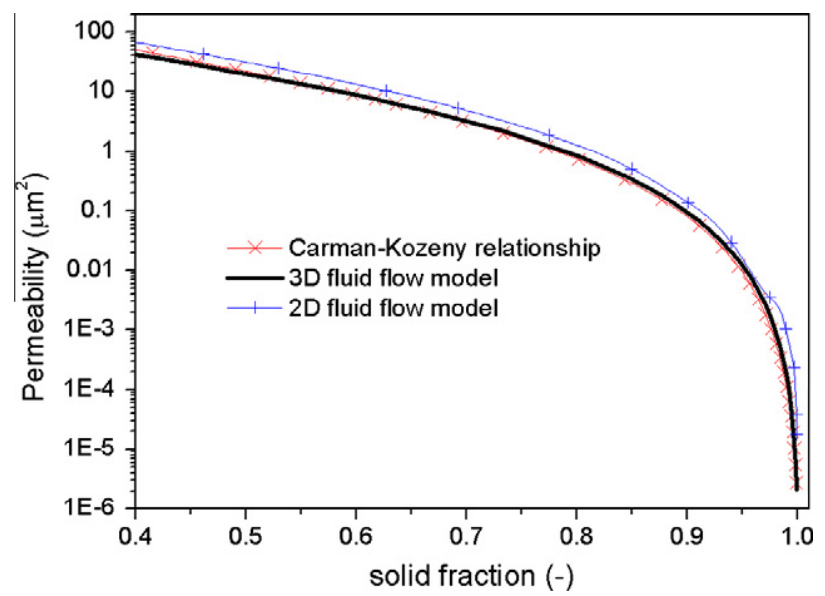

Fig. 5. The variation in permeability $\mathcal{K}$ with $g_{\mathrm{s}}$ for a random network of grains calculated using the 3-D fluid flow model. The 2-D fluid flow model of Vernède et al. [18] and the Carman-Kozeny relationship are also provided for comparison.

is no longer valid and the contribution of the flow at vortices, i.e. points where four grains meet, becomes also important. As can be seen, the permeability calculated with the 3-D fluid flow model follows the Carman-Kozeny relation closely until the very end of solidification with the accuracy improving with increasing $g_{\mathrm{s}}$. In contrast, the permeability of the 2-D fluid flow model is higher than the Carman-Kozeny predictions, a difference that can be explained by the smaller value of $A_{s \ell}$ for a 2-D assembly of grains compared with the 3-D case considered here [16]. Please note that this discrepancy does not appear in Vernède et al. [18] as the product $\mathcal{K} S_{\mathrm{v}}^{2}$, where $S_{\mathrm{v}}=A_{s \ell} / g_{\mathrm{s}}$, and not $\mathcal{K}$ was reported as a function of $g_{\mathrm{s}}$. While averaging methods, such as the Darcy equation, dictate that a lower permeability represents a higher average pressure drop, the local pressure drop predicted by the 2-D model is higher than the local pressure drop predicted by the 3$\mathrm{D}$ model. This is because the local pressure drop is closely linked to the actual number of channels available for feeding. a 2-D geometry liquid cannot pass once two grains have coalesced, whereas liquid can move around in the 3$\mathrm{D}$ model, due to the additional third dimension. As pointed out in Phillion et al. [16], both the solid and liquid phases can percolate in three dimensions, while this cannot be the case in two dimensions.

\subsubsection{Solidification shrinkage and deformation}

In the second test the volume of liquid required to feed solidification shrinkage and deformation is considered. The amount of liquid required to feed a deforming and solidifying isothermal RVE can be calculated analytically, and is given by:

$\left(\frac{Q}{V}\right)=\beta \frac{d g_{\mathrm{s}}}{d t}+\dot{\varepsilon}_{\mathrm{sv}}$

where $V$ is the volume of the RVE, and $Q$ is the volumetric flow $\left(\mathrm{m}^{3} \mathrm{~s}^{-1}\right)$. To simulate this case using the present 3-D fluid flow granular model all the surfaces of the RVE are

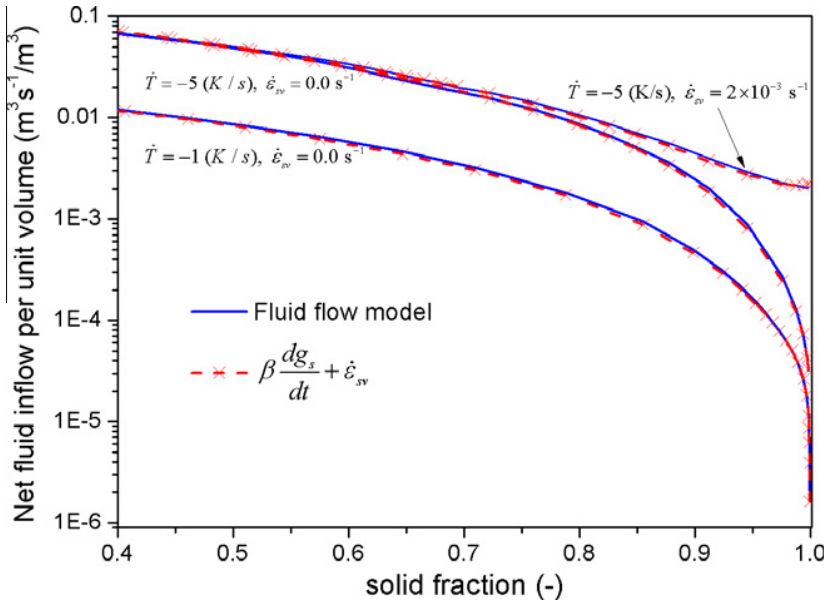

Fig. 6. A comparison of the variation in net fluid inflow per unit volume with $g_{\mathrm{s}}$ due to solidification shrinkage and semi-solid deformation as calculated using the 3-D fluid flow model and with Eq. (14) at two different cooling rates $\left(\dot{T}=-5\right.$ and $\left.-1 \mathrm{~K} \mathrm{~s}^{-1}\right)$ and two different strain rates $\left(\dot{\varepsilon}_{\mathrm{sv}}=0.0\right.$ and $\left.2 \times 10^{-3} \mathrm{~s}^{-1}\right)$.

closed except for one where feeding is allowed. Solidification and deformation is then initiated, and the volumetric liquid flow into the RVE through the open surface is recorded as a function of $g_{\mathrm{s}}$. Fig. 6 shows a comparison of the variation in net fluid inflow with $g_{\mathrm{s}}$ due to solidification shrinkage and semi-solid deformation as calculated using Eq. (14), and as calculated by the 3-D fluid flow model for two different cooling rates $\left(\dot{T}=-5\right.$ and $\left.-1 \mathrm{~K} \mathrm{~s}^{-1}\right)$ and two different strain rates $\left(\dot{\varepsilon}_{\mathrm{sv}}=0.0\right.$ and $\left.2 \times 10^{-3} \mathrm{~s}^{-1}\right)$. The evolution of $g_{\mathrm{s}}$ for this alloy was calculated using the granular solidification model [16]. As can be seen, there is good agreement for both cooling rates between the two curves. Hence the 3-D fluid flow model is able to accurately simulate the liquid flow induced by solidification shrinkage and semi-solid deformation. The formation of hot tears is similar to porosity formation in the sense that it is linked to a lack of liquid feeding in the mushy zone, but additionally requires shear or tensile deformation. As can be seen in Fig. 6, although the need for fluid to account for solidification shrinkage decreases with increasing $g_{\mathrm{s}}$, the need for fluid to account for deformation remains nearly constant at high $g_{\mathrm{s}}$. Previous work has also shown that, locally, the fluid needed to counteract deformation may even increase at high $g_{\mathrm{s}}$ since the deformation localizes along a few preferential paths [25].

\subsection{Hot tearing sensitivity index}

Although many hot tearing criteria have been suggested in the literature (review by Eskin et al. [30] for example), the pressure drop due to shrinkage and deformation is a key parameter of the most recently developed criteria. In the RDG criterion [4] the amount of liquid $Q$ needed to feed a mushy zone under deformation is given by Eq. (14).

Assuming an isothermal mushy zone under uniform straining of the solid skeleton, the pressure drop or pres- 
sure gradient according to Darcy's law is thus proportional to:

$$
\Delta p \propto\left(\frac{\beta \dot{g}_{s}+\dot{\varepsilon}_{\mathrm{sv}}}{\mathcal{K}}\right)
$$

Therefore the coefficient $M=\left(\frac{\beta \dot{g}_{\mathrm{s}}+\dot{\boldsymbol{\varepsilon}}_{\mathrm{sv}}}{\mathcal{K}}\right)$ represents the sensitivity of the mushy zone to hot tearing.

The expression $M$ has been computed using the simulations performed for Figs. 5 and 6. The results are shown in Fig. 7 as a function of $g_{\text {s }}$ at a cooling rate of $\dot{T}=-1 \mathrm{~K} \mathrm{~s}^{-1}$ for different strain rates between $\dot{\varepsilon}_{\mathrm{sv}}=0$ and 0.01 . As can be seen, $M$ values at $g_{\mathrm{s}}<0.9$ are insignificant, but increase sharply beginning at $g_{\mathrm{s}}=0.9$; with increasing strain rate the rapid increase in $M$ occurs at lower $g_{\mathrm{s}}$. As shown by many previous authors (see, for example, Davidson et al. [31]), hot tearing susceptibility is significantly increased at $g_{\mathrm{s}}>0.9$. These results confirm those findings, and indicate that it is the feeding ability of the mushy zone that determines hot tear formation. At values of $g_{s}>0.9$ the feeding ability becomes significantly reduced. The addition of the strain rate term, i.e. a deforming mushy zone, simply enhances the problem of feeding and enables the crack to form and grow at an earlier stage.

\subsection{Case study: direct chill casting of round billets}

The direct chill (DC) semi-continuous casting process[32], schematically presented in Fig. 8, is widely used in the aluminum alloy industry. Experimentally, hot tears are observed in the center of round billets when the casting speed is increased [32]. To examine fluid flow in DC casting in the context of hot tearing a column of semi-solid metal located at the center of the billet and spanning the distance between the solidus and liquidus temperatures has been studied using the new 3-D granular fluid flow model. In this region lateral feeding is assumed to be negligible for axisymmetric reasons. The characteristics of the primary and secondary cooling systems, such as the amount and quality

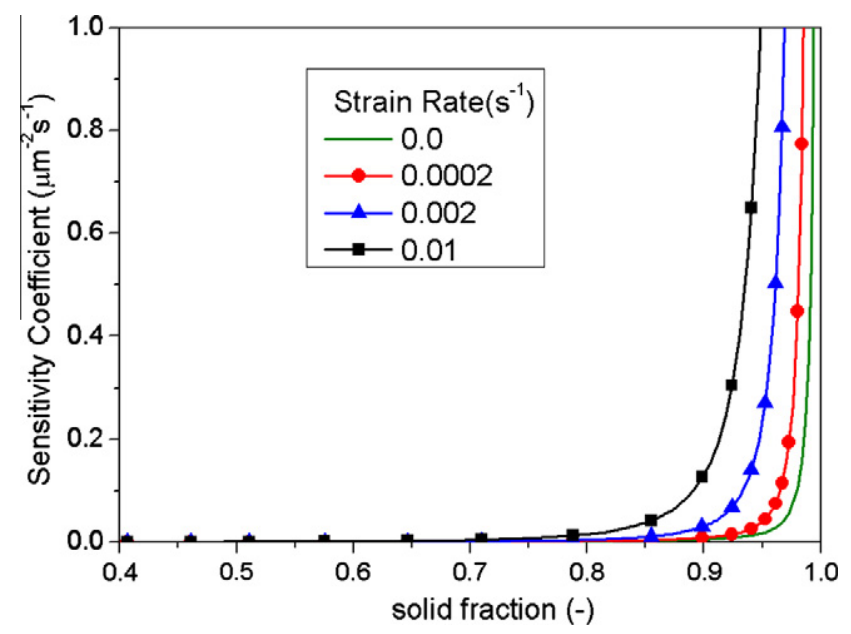

Fig. 7. Variation in hot tearing sensitive coefficient $M$, i.e. $\left[\left(\beta \dot{g} \mathrm{~s}+\dot{\varepsilon}_{\mathrm{sv}}\right) / \mathcal{K}\right]$, with $g_{\mathrm{s}}$ for four different strain rates. of the cooling water and the mold geometry, together with the casting speed, determine the sump depth $h$ and cooling rate $\dot{T}$. The typical values of $h$ and $\dot{T}$ for the billet of $200 \mathrm{~mm}$ diameter considered in this study are $100 \mathrm{~mm}$ and $5 \mathrm{~K} \mathrm{~s}^{-1}$ at a casting speed of $90 \mathrm{~mm} \mathrm{~min}^{-1}$ (i.e. a vertical thermal gradient of $3.33 \times 10^{3} \mathrm{~K} \mathrm{~m}^{-1}$ ) [32]. With this thermal history the evolution of $g_{\mathrm{s}}$ at any point along the billet center line can be computed using the granular solidification model [16]. The resulting semi-solid geometry will be used to calculate the pressure drop associated with solidification shrinkage and semi-solid deformation under a uniform volumetric strain rate $\dot{\varepsilon}_{\mathrm{sv}}$.

In a way similar to heavy computations performed on multiple processors the semi-solid column is sub-divided into six smaller RVEs, each containing 16,000 $(40 \times 20 \times 20)$ grains, in order to reduce the computational cost, as shown in Fig. 8. The size of each RVE is $H=4 \mathrm{~mm}$ along the axis of the billet and $2 \times 2 \mathrm{~mm}$ in cross-section. Since the values of the fluxes $Q_{\mathrm{ij}}$ exchanged at the boundary between the RVE domains (i) and $(i+1)(i=1-6)$ are unknown the simulation is performed twice. Please note that since $Q_{\mathrm{ij}}$ depends only on the pressure gradient, the actual pressure value imposed is not critical to the flux computation. For the first simulation the $Q_{\mathrm{ij}}$ values are calculated sequentially in order to acquire the fluid flow within the mushy zone. The sequential calculation begins with the first $\operatorname{RVE}(i=1)$ located at the bottom of the mushy zone. Since there is no flux possible at the bottom boundary (i.e. at the interface with the fully solid material) the simulation can be performed by imposing a constant

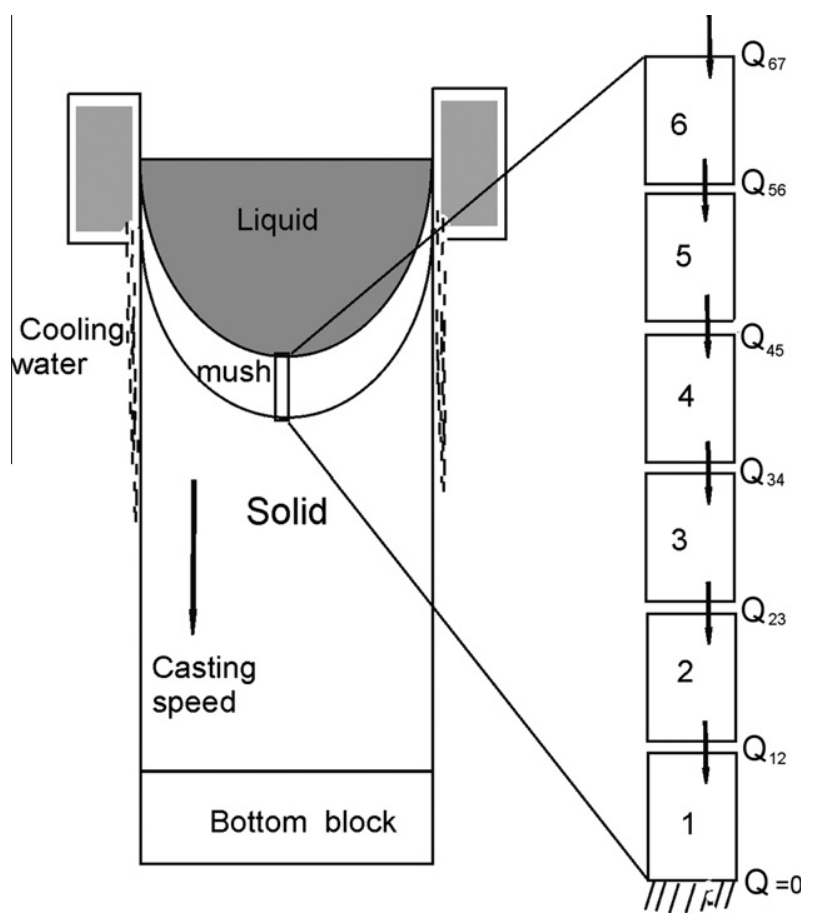

Fig. 8. A schematic diagram illustrating the DC casting process for round billets. As shown on the right, the mushy zone has been subdivided into six segments in order to simulate fluid flow within the sump region. 


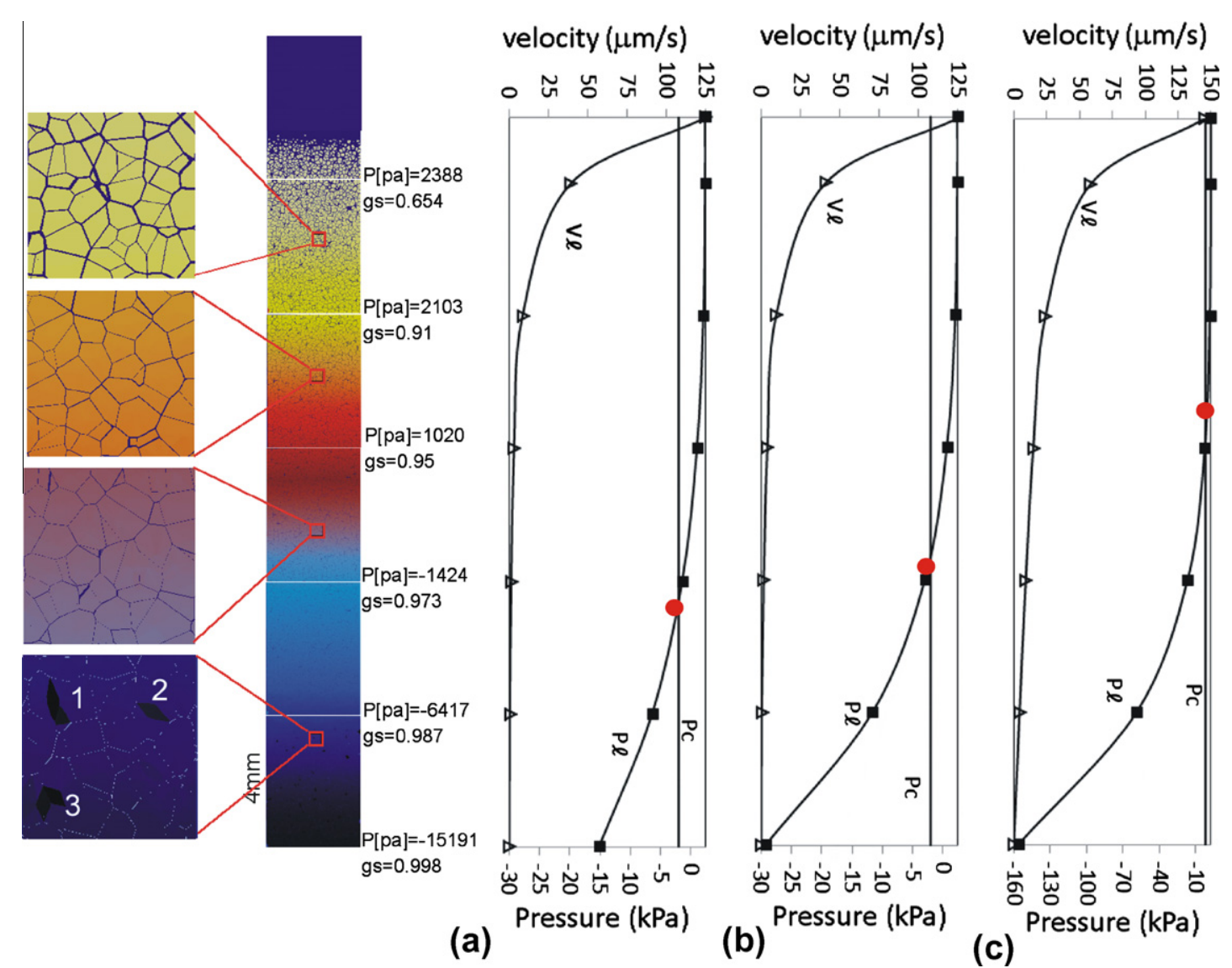

Fig. 9. The calculated pressure drop through a mushy zone located at the center of a solidifying aluminum alloy $\mathrm{DC}$ cast round billet for an Al-1 wt.\% $\mathrm{Cu}$ alloy cooled at $-5 \mathrm{~K} \mathrm{~s}^{-1}$ in a gradient of $3.33 \times 10^{3} \mathrm{~K} \mathrm{~m}^{-1}$ at strain rates of (a) $\dot{\varepsilon}_{\mathrm{sv}}=0.0$, (b) $10^{-4}$ and (c) $10^{-3} \mathrm{~s}$. $P_{\mathrm{a}}$ has been set to 0 in this case. The grain view and flow shown on the left corresponds to case (a).

pressure equal to zero at the upper boundary. This allows one to calculate the entering flow $Q_{12}$ at this upper boundary, since the evolution of $g_{\mathrm{s}}$ is known from the thermal field. This value is then imposed on the bottom side of the second $\operatorname{RVE}(i=2)$ and a constant pressure is imposed on the upper side. The flow $Q_{23}$ is thus obtained and the problem is repeated sequentially up to the sixth RVE. For the second simulation the process is reversed in order to acquire the actual pressure drop within each RVE. This time the simulation begins with the RVE $i=6$, since the pressure on the upper side of this volume $\left(p_{\mathrm{a}}+\rho g h\right)$ is known, where $p_{\mathrm{a}}$ is the atmospheric pressure. This pressure is imposed on the upper side of this RVE, while the flow $Q_{56}$ calculated from the first simulation is imposed at the bottom boundary. With these two conditions, as for Fig. 5 , the actual pressure at the surface $S_{56}$, i.e. $p_{56}$, can be computed using the granular fluid flow model. This process is continued until the pressure drop within each RVE is acquired.

Fig. 9 shows the calculated pressure drop through the center line mushy zone of a DC cast billet along with the associated Darcy's velocity. The pressure drop is entirely due to solidification shrinkage and semi-solid deformation at uniform and fixed volumetric strain rates of $\dot{\varepsilon}_{\mathrm{sv}}=0.0$
(Fig. 9a), $10^{-4}$ (Fig. 9b) and $10^{-3} \mathrm{~s}^{-1}$ (Fig. 9c). Although the minimum average pressures reported are approximately $-15 \mathrm{kPa}$ (Fig. 9a), $-29 \mathrm{kPa}$ (Fig. 9b) and $-156 \mathrm{kPa}$ (Fig. 9c), the minimum calculated local pressure is typically much lower, $-459 \mathrm{kPa}$ (Fig. 9a), $-498 \mathrm{kPa}$ (Fig. 9b) and $-795 \mathrm{kPa}$ (Fig. 9c), in some of the channels which are recognizable as dark spots (noted as 1,2,3 in Fig. 9, RVE $i=1$ ). These extremely large pressure drops occur due to the low feeding ability of some individual thin channels. However, in a granular model that includes additional physical phenomena, including porosity formation and solid grain deformation/displacement [23], such a high depression will be lessened. In this case, when the pressure within the thin liquid channels falls below a cavitation pressure $\left(p_{\mathrm{c}}\right)$ a void would form, giving rise to a crack [4]. Since the radius of curvature of the pores is also dictated by the width of the liquid channel, the cavitation pressure itself also depends on the thickness of the liquid channel. As shown in Fig. 9, if $p_{\mathrm{c}}$ is set to $2 \mathrm{kPa}$ for the sake of simplicity [4,32], hot tearing occurs at $g_{\mathrm{s}}=0.979$ (Fig. 9a), 0.964 (Fig. 9b) and 0.938 (Fig. 9c). Therefore, with increasing $\dot{\varepsilon}$ the hot tearing occurs at lower $g_{\mathrm{s}}$ and hot tearing susceptibility is significantly increased. This remark was also deduced previously from the sensitivity coefficient $M$. 


\section{Conclusions}

A 3-D granular fluid flow model has been developed to simulate the localization of feeding through the mushy zone. In this new model, the pressure drop was calculated assuming Poiseuille flow in each channel. The model predictions (permeability and shrinkage) were validated against the results obtained from averaging techniques. The results show that the model is able to calculate the permeability of the mushy zone and the flow associated with solidification shrinkage accurately for values of $g_{\mathrm{s}}$ greater than 0.4. Although the average values fit well with those obtained by simple averaging techniques such as the Carman-Kozeny model it is also shown that locally, in particular in the thinnest channels of liquid, the pressure drop can deviate substantially from the analytical solution.

A hot tearing criterion has been developed for globular solidification and the sensitivity of the mushy zone to hot tearing at various $g_{\mathrm{s}}$ values has been analyzed using the results obtained with the fluid flow model. Although the hot tearing sensitivity coefficient at $g_{\mathrm{s}}<0.9$ has an insignificant value, it rapidly increases above $g_{\mathrm{s}}=0.9$. This rapid increase confirms that the susceptibility of the mushy zone to hot tearing occurs at a high solid fraction and near the end of solidification due to its low feeding ability. Finally, the model has been applied to the particular case of DC casting of an aluminum extrusion billet. The calculated pressure demonstrates that deep within the mushy zone, where the permeability is low, the local pressure can be very low in the thinnest liquid channels, even though the average pressure drop remains limited. This low pressure in such channels will have to be placed into relation to the cavitation pressure required to nucleate and grow a non-wetting pore at those locations. Furthermore, this fluid flow model is being coupled with a semi-solid deformation model [23] by the present authors. The hydromechanical coupling will provide a fairly unique tool and good insight into the determinant phenomena of hot tearing and, in particular, into the contribution of localization of strain and feeding.

\section{Acknowledgements}

The authors would like to thank the Swiss Competence Centre for Materials Science and Technology, and partner companies within the thematic area "Multi-scale, multiphenomena modeling of metallic systems" for funding this research. We would like also to thank Jean-Luc Desbiolles for writing a portion of the GMS-3D code.

\section{References}

[1] Dantzig JA, Rappaz M. Solidification. Lausanne: EPFL Press; 2009.

[2] Feurer U. Giessereiforschung 1976;28:75.

[3] Niyama E. US joint seminar on solidification of metals and alloys. Tokyo: Japan Society for the Promotion of Science; 1977. p. 271.

[4] Rappaz M, Drezet JM, Gremaud M. Metall Trans A 1999;30:449.

[5] Farup I, Drezet JM, Rappaz M. Acta Mater 2001;49:1261.

[6] Terzi S, Salvo L, Suery M, Limodin N, Adrien J, Maire E, et al. Scripta Mater 2009;61:449.

[7] Ludwig O, Drezet JM, Martin C, Suéry M. Metall Trans A 2005;36: 1525.

[8] Mathier V, Vernède S, Jarry P, Rappaz M. Metall Trans A 2009;40: 943.

[9] M'Hamdi M, Mo A, Fjaer HG. Metall Mater Trans A: Phys Metall Mater Sci 2006;37A:3069.

[10] Mo A, M'Hamdi M, Fjaer HG. In: Stefanescu DM, Warren J, Jolly M, Krane M, editors. Modeling of casting, welding and advanced solidification processes-X. Warrendale (PA): TMS; 2003. p. 199.

[11] Monroe CA, Beckermann C, Klinkhammer J. In: Cockcroft SL, Maijer DM, editors. Modeling of casting, welding, and advanced solidification processes, XII. Warrendale (PA): TMS; 2009.

[12] Carman P. Trans Inst Chem Eng 1937;15:150.

[13] Carman P. J Soc Chem Ind 1938;57:225.

[14] Mathier V, Jacot A, Rappaz M. Model Simul Mater Sci Eng 2004;12: 479.

[15] Vernède S, Rappaz M. Acta Mater 2007;55:1703.

[16] Phillion AB, Desbiolles JL, Rappaz M. In: Cockcroft S, Maijer D, editors. Modeling of casting, welding, and advanced solidification processes XII. Warrendale (PA): TMS; 2009. p. 353.

[17] Dijkstra WO, Vuik C, Dammers AJ, Katgerman L. In: Rappaz M, Beckerman C, Trivedi R, editors. Solidification processes and Microstructures: a symposium in honor of Wilfried Kurz. Charlotte (NC): TMS; 2004. p. 151.

[18] Vernède S, Jarry P, Rappaz M. Acta Mater 2006;54:4023.

[19] Phillion AB, Vernede S, Rappaz M, Cockcroft SL, Lee PD. Int J Cast Met Res 2009;22:240.

[20] Phillion AB, Cockcroft SL, Lee PD. Acta Mater 2008;56:4328.

[21] Vernède S, Dantzig JA, Rappaz M. Acta Mater 2009;57:1554.

[22] Phillion AB, Cockcroft SL, Lee PD. Model Simul Mater Sci Eng 2009; 17:055011.

[23] Sistaninia M, Phillion AB, Drezet JM, Rappaz M. Metall Trans A 2011;42:239.

[24] Rappaz M, Jacot A, Boettinger WJ. Metall Trans A 2003;34:467.

[25] Charbon C, Rappaz M. Acta Mater 1996;44:2663.

[26] Drezet J-M, Sistaninia M, Rappaz M. Matériaux Tech 2010;98:261.

[27] Dongarra J, Lumsdaine R, Pozo R, Remington K. In: Proceedings of the second object oriented numerics conference. Sunriver, Oregon; 1992. p. 214.

[28] Bernard D, Nielsen O, Salvo L, Cloetens P. Mater Sci Eng A: Struct Mater Prop Microstruct Process 2005;392:112.

[29] Khajeh E, Maijer DM. Acta Mater 2010;58:6334.

[30] Eskin DG, Suyitno, Katgerman L. Prog Mater Sci 2004;49:629.

[31] Davidson C, Viano D, Lu L, St. John D. Int J Cast Met Res 2006;19: 59.

[32] Drezet JM, Rappaz M. In: Whiteley PR, Grandfield JF, editors. Sixth Australian Asian pacific conference on aluminium cast house technology. Warrendale (PA): Minerals, Metals \& Materials Society; 1999. 